Ann. Biol. anim. Bioch. Biophys., 1977, 17 (1), 85-93.

\title{
Influence sur l'aminoacidémie de la perfusion intraveineuse de méthionine à des chèvres laitières
}

\author{
par C. CHAMPREDON, R. PION et J. PRUGNAUD \\ avec la collaboration technique de Françoise BARRE, G. BAYLE et A. SELLE \\ Laboratoire d'Efude du Méfabolisme Azoté, I. N. R. A., \\ Theix, Saint-Genes-Chompanelle, 63110 Beaumont
}

Summary. Effect of intravenous methionine perfusion on amino acid levels in dairy goats.

Three dairy goats are fed hay and a concentrate containing mainly barley ; salines containing constant levels of valine, isoleucine, leucine and histidine, as well as graded levels of methionine are then perfused intravenously (tables 1,2). The solutions are perfused continuously during eight 7-day periods. The ist period (saline only), and the 9ih and 10th (without perfusion) are control periods. The amounts ingested and milk production are recorded daily; milk fat and protein content are measured at the end of each period. Blood is sampled daily to estimate urea and blood sugar levels and at the end of each period to evaluate free amino acids.

Perfusion has no effect on food intake and milk production. Milk protein content increases during the experiment (table 3). Blood free methionine level augments with the amount of methionine perfused ; a direct relation between blood methionine level and the « uptakesupply balance $\gg$ is demonstrated (table 4). Branched-chain amino acid concentrations increase with perfusion (fig. 1), but they decrease in the two goats producing less milk when more than $2 \mathrm{~g}$ per day of methionine is perfused. The relative blood level increase with perfusion is lower for histidine than for branched-chain amino acids. Threonine and phenylalanine levels seem slightly lower with increasing amounts of perfused methionine. In our conditions, methionine is the first limiting amino acid for lactating goats and threonine, valine, isoleucine, leucine and histidine seem to be secondarily limiting.

\section{Introduction.}

La première étape de la détermination du besoin en acides aminés des ruminants est l'identification des composés qui sont les plus limitants. Une des méthodes utilisables consiste à mesurer les teneurs en acides aminés libres du sang ou du plasma de ruminants recevant des protéines ou des acides aminés par infusions postruminales (Derrig, Clark et Davis, 1974. Spires ef al., 1975 ; Clark, 1975) ou des acides aminés par voie intraveineuse (Fisher, 1972 ; Champredon, 1972). En effet, l'importance des 
fermentations qui se produisent dans le rumen ne permet pas de faire varier de manière connue par l'alimentation les proportions des différents acides aminés qui seront absorbés dans l'intestin (Clarke, Ellinger et Phillipson, 1966 ; Champredon, Pion et Thivend, 1971), sauf par l'ingestion de protéines arrivant directement dans l'intestin (Chalupa, 1975), grâce à des traitements technologiques leur permettant de résister à l'attaque microbienne dans le rumen (Ferguson, Hemsley et Reis, 1967 ; Leroy, Zelter et François, 1964). Le mélange d'acides aminés mis à la disposition des tissus des ruminants risque dans de nombreux cas de comporter des proportions trop faibles de certains composés dont la méthionine.

Le but de cette expérience est d'étudier les effets de l'infusion intraveineuse de doses croissanfes de méthionine à des chèvres en milieu de lactation sur leur production laitière ef sur l'évolution des teneurs dans le sang des autres acides aminés indispensables.

\section{Matériel et méthodes.}

Trois chèvres en milieu de lactation (nos 11, 15 et 16) placées dans des cages à digestibilité, recevaient du foin de prairie naturelle de qualité moyenne et un aliment concentré à base d'orge (70 p. 100) contenant 20 p. 100 de farine de luzerne déshydratée, 5 p. 100 de mélasse de canne et 5 p. 100 d'un mélange minéral et vitaminique (Sanders-Bovian 16-16). Après deux semaines d'adaptation, un cathéter a été posé dans la veine jugulaire ef une solution aqueuse stérile de chlorure de sodium à 0,9 p. 100 (période 1) a été perfusée en continu à chaque chèvre au moyen d'une pompe péristaltique pendant 7 jours, à raison de $250 \mathrm{ml}$ environ par $24 \mathrm{~h}$ (tabl. 1). Durant

TABLEAU 1

Débit des solutions * perfusées ( $\mathrm{ml}$ par 24 heures)

\begin{tabular}{|c|c|c|c|c|c|c|c|c|}
\hline Périodes...... & 1 & 2 & 3 & 4 & 5 & 6 & 7 & 8 \\
\hline $\begin{array}{l}n^{0} 11 \ldots \ldots \ldots \\
n^{0} 15 \ldots \ldots \ldots \\
n^{0} 16 \ldots \ldots \ldots\end{array}$ & $\begin{array}{l}276 \\
276 \\
250\end{array}$ & $\begin{array}{l}255 \\
240 \\
223\end{array}$ & $\begin{array}{l}235 \\
223 \\
222\end{array}$ & $\begin{array}{l}262 \\
245 \\
241\end{array}$ & $\begin{array}{l}178 \\
170 \\
175\end{array}$ & $\begin{array}{l}300 \\
290 \\
293\end{array}$ & $\begin{array}{l}296 \\
295 \\
294\end{array}$ & $\begin{array}{l}267 \\
267 \\
263\end{array}$ \\
\hline
\end{tabular}

* Composition des solutions (en g par litre) :

- En période 1 : Chlorure de sodium : 9,00 .

- En période 2 d̀ 8 : Chlorure de sodium : 9,00; L-valine : 6,67 ; L-isoleucine : 3,75; L-leucine : 7,00 ; L-histidine base : 3,00.

Les teneurs des solutions en L-méthionine ont varié de 0 à $8,55 \mathrm{~g}$ par litre.

la semaine suivante (période 2), la solution perfusée contenait de la L-valine $(1,7 \mathrm{~g}$ par jour environ), de la L-leucine (1,8 g par jour), de la L-isoleucine (1, $0 \mathrm{~g}$ par jour) et de la L-histidine $(0,8 \mathrm{~g}$ par jour). Durant les périodes 3 à 8 d'une semaine chacune, des quantités voisines de ces mêmes acides aminés étaient perfusées et des quantités 
croissantes de L-méthionine étaient ajoutées aux solutions (łabl. 2). L'expérience s'est terminée par deux périodes de 7 jours durant lesquelles les animaux ont été maintenus en cages sans qu'aucune solution ne leur soit administrée par voie intraveineuse.

TABLEAU 2

Quantifés de L-méthionine apportées por les perfusions (g par jour)

\begin{tabular}{lllllllll}
\hline Périodes $\ldots \ldots \ldots$ & 1 & 2 & 3 & 4 & 5 & 6 & 7 & 8 \\
\hline & & & & & & & & \\
\hline
\end{tabular}

Les quantités d'aliments consommées par les animaux ainsi que le lait produit à chacune des deux traites ont été mesurés chaque jour. Le taux butyreux et le taux de matières azotées ont été déterminés sur un échantillon moyen du lait produit au cours des 4 dernières traites de chacune des 10 périodes expérimentales. Des prélèvements de sang jugulaire ont été effectués presque quotidiennement $5 \mathrm{~h}$ après la distribution du repas du matin afin d'observer l'évolution au cours de l'expérience de la glycémie et de l'urémie. Celles-ci étaient mesurées respectivement par les méthodes à la glucose oxydase et à la diacétylmonoxime. Les teneurs en acides aminés libres du sang étaient déterminées après extraction éthanolique à partir d'échantillons prélevés en fin de périodes aux heures précédemment indiquées, par chromatographie sur résines échangeuses d'ions au moyen d'un appareil automatique (Pawlak et Pion, 1968).

Le débit de la pompe de perfusion a été réduif durant la période 5 par suite d'une défaillance du mécanisme ; le traitement des échantillons destinés à la détermination de l'aminoacidémie libre n'a donc pas été effectué. Il en a été de même en ce qui concerne les échantillons prélevés à la fin de périodes au cours desquelles des incidents d'ordre sanitaire ou technique préjudiciables à la validité des résultats se sont produits (période 3 , chèvres nos 11 et 16 , période 6 , chèvre no 15 ; période 7 , chèvre $\left.n^{\circ} 16\right)$.

\section{Résultats.}

Les animaux ont bien supporté leur séjour en cage à digestibilité. La perfusion d'acides aminés n'a pas eu d'influence notable sur l'appétit des animaux ni sur l'évolution de leur production laitière, sauf en ce qui concerne la chèvre $n^{0} 11$ entre les périodes 1 et 2 (tabl. 3). Le taux butyreux du lait des 3 animaux diminue de manière importante entre la fin de la période 1 et la fin de la période 2 puis, varie relativement peu pour les chèvres $n^{\text {os }} 11$ el 15 . Par contre, des valeurs assez différentes les 
unes des autres ont été observées dans le cas de l'animal no 16 pendant les 5 premières périodes.

Le taux de matières azotées du lait (tabl. 3) a évolué de la même manière pour les trois chèvres durant toute l'expérience ; voisin de 26 p. 100 en fin de première période, il était de 23,6 en deuxième période et de 26,6 en troisième période. Il a continué ensuite à s'élever pour être voisin de 32 en fin d'expérience. De ce fait, les quantités

TABLEAU 3

Quantités d'aliments ingérées ef productions laitières des chèvres

\begin{tabular}{|c|c|c|c|c|c|c|c|c|c|c|}
\hline Périodes & 1 & 2 & 3 & 4 & 5 & 6 & 7 & 8 & 9 & 10 \\
\hline \multicolumn{11}{|c|}{ Foin ingéré ( $\mathrm{kg}$ par jour) } \\
\hline $\begin{array}{l}\text { No } 11 \\
\text { No } 15 \ldots \ldots \ldots \\
\text { No } 16 \ldots \ldots \ldots \ldots\end{array}$ & $\begin{array}{l}1,10 \\
1,60 \\
1,25\end{array}$ & $\begin{array}{l}0,90 \\
2,00 \\
1,20\end{array}$ & $\begin{array}{l}0,95 \\
1,30 \\
1,10\end{array}$ & $\begin{array}{l}1,10 \\
1,20 \\
0,95\end{array}$ & $\begin{array}{l}1,35 \\
1,30 \\
1,05\end{array}$ & $\begin{array}{l}1,50 \\
1,70 \\
1,00\end{array}$ & $\begin{array}{l}1,60 \\
1,60 \\
1,25\end{array}$ & $\begin{array}{l}1,40 \\
1,25 \\
0,75\end{array}$ & $\begin{array}{l}1,50 \\
1,60 \\
0,70\end{array}$ & $\begin{array}{l}1,60 \\
1,65 \\
0,60\end{array}$ \\
\hline \multicolumn{11}{|c|}{ Aliment concentré ingéré ( $\mathrm{kg}$ par jour) } \\
\hline $\begin{array}{l}\text { No } 11 \\
\text { No } 11 \\
\text { No } 15 \\
\text { No } 16 \ldots \ldots \ldots\end{array}$ & $\begin{array}{l}1,50 \\
1,80 \\
1,30\end{array}$ & $\begin{array}{l}1,00 \\
2,00 \\
1,50\end{array}$ & $\begin{array}{l}1,00 \\
1,95 \\
1,50\end{array}$ & $\begin{array}{l}1,00 \\
2,00 \\
1,50\end{array}$ & $\begin{array}{l}1,00 \\
1,80 \\
1,50\end{array}$ & $\begin{array}{l}1,00 \\
1,70 \\
1,40\end{array}$ & $\begin{array}{l}1,25 \\
1,80 \\
1,25\end{array}$ & $\begin{array}{l}1,25 \\
1,90 \\
1,45\end{array}$ & $\begin{array}{l}1,25 \\
2,00 \\
1,50\end{array}$ & $\begin{array}{l}1,25 \\
2,00 \\
1,50\end{array}$ \\
\hline \multicolumn{11}{|c|}{ Production laitière (kg par jour) } \\
\hline $\begin{array}{l}\text { No } 11 \\
\text { No } 11 \\
\text { No } 15 \ldots \ldots \ldots \\
\text { No }^{0} 16 \ldots \ldots \ldots \ldots\end{array}$ & $\begin{array}{l}3,00 \\
4,20 \\
3,00\end{array}$ & $\begin{array}{l}2,00 \\
4,10 \\
2,80\end{array}$ & $\begin{array}{l}2,00 \\
3,70 \\
2,70\end{array}$ & $\begin{array}{l}1,90 \\
3,70 \\
2,70\end{array}$ & $\begin{array}{l}1,85 \\
3,20 \\
2,40\end{array}$ & $\begin{array}{l}1,75 \\
3,20 \\
2,60\end{array}$ & $\begin{array}{l}1,90 \\
3,10 \\
2,00\end{array}$ & $\begin{array}{l}2,10 \\
3,10 \\
2,05\end{array}$ & $\begin{array}{l}2,20 \\
3,00 \\
1,85\end{array}$ & $\begin{array}{l}2,00 \\
2,85 \\
1,65\end{array}$ \\
\hline \multicolumn{11}{|c|}{ Taux azoté du lait (g p. 1000 ) } \\
\hline $\begin{array}{l}\text { No } 11 \\
\text { No } 11 \\
\text { No } 15 \\
\text { No } 16\end{array}$ & $\begin{array}{l}26,4 \\
24,8 \\
26,6\end{array}$ & $\begin{array}{l}21,8 \\
23,7 \\
25,3\end{array}$ & $\begin{array}{l}24,9 \\
27,7 \\
27,3\end{array}$ & $\begin{array}{l}25,8 \\
26,3 \\
27,3\end{array}$ & $\begin{array}{l}26,0 \\
25,6 \\
26,5\end{array}$ & $\begin{array}{l}28,2 \\
27,5 \\
26,6\end{array}$ & $\begin{array}{l}27,8 \\
28,9 \\
28,8\end{array}$ & $\begin{array}{l}28,3 \\
29,7 \\
29,6\end{array}$ & $\begin{array}{l}31,3 \\
32,6 \\
31,4\end{array}$ & \\
\hline \multicolumn{11}{|c|}{ Taux butyreux du lait (g p. 1000 ) } \\
\hline $\begin{array}{l}\text { No } 11, \ldots \ldots \ldots \\
\text { No } 15 \ldots \ldots \ldots \\
\text { No } 16 \ldots \ldots \ldots\end{array}$ & $\begin{array}{l}32 \\
35 \\
32\end{array}$ & $\begin{array}{l}22 \\
23 \\
25\end{array}$ & $\begin{array}{l}36 \\
33 \\
37\end{array}$ & $\begin{array}{l}32 \\
29 \\
25\end{array}$ & $\begin{array}{l}31 \\
27 \\
31\end{array}$ & $\begin{array}{l}33 \\
30 \\
26\end{array}$ & $\begin{array}{l}33 \\
30 \\
28\end{array}$ & $\begin{array}{l}30 \\
34 \\
28\end{array}$ & $\begin{array}{l}32 \\
37 \\
33\end{array}$ & \\
\hline
\end{tabular}

journalières de matières azotées excrétées par les chèvres dans le lait produit durant les derniers jours de chaque période ont diminué moins rapidement que la production laitière. La diminution est faible pour la chèvre $n^{0} 15$, alors que les valeurs obtenues augmentent à partir de la $2^{\mathrm{e}}$ période dans le cas de la chèvre $\mathrm{n}^{0} 11$.

Les urémies des chèvres sont généralement comprises entre 10 et $30 \mathrm{mg}$ pour $100 \mathrm{ml}$ de sang. Des valeurs comprises entre 30 et $50 \mathrm{mg}$ pour $100 \mathrm{ml}$ sont observées durant les périodes $n^{\circ} 5$ à 8 pour la chèvre $n^{\circ} 15$ et après l'arrêt de la perfusion dans le cas de l'animal $n^{0} 11$. La glycémie de la chèvre no 11 est comprise entre 40 et $75 \mathrm{mg}$ pour $100 \mathrm{ml}$ de sang; des valeurs comprises entre 50 et $80 \mathrm{mg}$ ef entre 50 et $90 \mathrm{mg}$ 
sont observées respectivement pour les animaux nos 15 et 16. C'est dans le sang de ce dernier que les variations d'un prélèvement à l'autre sont les plus importantes.

Les teneurs en méthionine (tabl. 4) varient relativement peu entre les périodes 1 et 2 sauf dans le cas de la chèvre no 16 où une nette diminution est constatée à la suite de l'infusion de la première solution comportant des acides aminés. Les teneurs

\section{TABLEAU 4}

Teneurs en méthionine libre du sang des chèvres ef « bilans apports-utilisations » en méthionine*

\begin{tabular}{|c|c|c|c|c|c|c|c|c|c|c|}
\hline Périodes & 1 & 2 & 3 & 4 & 5 & 6 & 7 & 8 & 9 & 10 \\
\hline \multicolumn{11}{|c|}{ Teneurs en méthionine (mg p. $100 \mathrm{~g}$ de sang) } \\
\hline $\begin{array}{l}\text { No } 11 \ldots \ldots \\
\text { No } 15 \ldots \ldots \\
\text { No } 16 \ldots \ldots\end{array}$ & $\begin{array}{l}0,64 \\
0,79 \\
0,92\end{array}$ & $\begin{array}{l}0,67 \\
0,73 \\
0,69\end{array}$ & 1,02 & $\begin{array}{l}1,06 \\
1,04 \\
0,99\end{array}$ & & $\begin{array}{l}1,59 \\
1,56\end{array}$ & $\begin{array}{l}1,54 \\
1,34\end{array}$ & $\begin{array}{l}1,30 \\
1,35 \\
1,52\end{array}$ & $\begin{array}{l}0,71 \\
0,82 \\
0,92\end{array}$ & $\begin{array}{l}0,73 \\
0,78 \\
0,72\end{array}$ \\
\hline \multicolumn{11}{|c|}{ «Bilans apports-utilisations » (g par jour) } \\
\hline $\begin{array}{l}\text { No } 11 \ldots \ldots \\
\text { No } 15 \ldots \ldots \\
\text { No } 16 \ldots \ldots\end{array}$ & $\begin{array}{l}0,83 \\
0,98 \\
0,67\end{array}$ & $\begin{array}{l}0,91 \\
1,73 \\
1,12\end{array}$ & 1,32 & $\begin{array}{l}1,72 \\
1,83 \\
1,57\end{array}$ & & $\begin{array}{l}3,03 \\
2,62\end{array}$ & $\begin{array}{l}4,08 \\
3,87\end{array}$ & $\begin{array}{l}3,46 \\
3,37 \\
3,14\end{array}$ & $\begin{array}{l}1,07 \\
1,40 \\
1,04\end{array}$ & $\begin{array}{l}1,31 \\
1,56 \\
1,12\end{array}$ \\
\hline
\end{tabular}

* « Bilans apports-utilisations »= somme des apports de méthionine diminuée de la quantité de méthionine utilisée par la glande mammaire (Champredon, 1972).

La somme des apports de méthionine est estimée à partir de la teneur en ce composé des matières azotées arrivant dans l'intestin et des quantités de matières azotées digestibles ingérées ; on y ajoute éventuellement les quantités apportées par les perfusions.

Les utilisations sont estimées à partir des quantités de méthionine contenues dans le lait produit par les chèvres.

augmentent généralement de la période 2 aux périodes 6 ou 7 ; elles sont parfois légèrement plus faibles en période 8 qu'en période 6 ou 7 ; elles redeviennent proches en périodes 9 et 10 de ce qu'elles étaient en début d'expérience.

Les teneurs en acides aminés à chaîne ramifiée (fig. 1) sont plus élevées durant les périodes au cours desquelles ces composés sont apportés par voie intraveineuse que lorsqu'ils ne sont pas perfusés, sauf en ce qui concerne la $8^{\mathrm{e}}$ et dans certains cas la 7 e période où les valeurs observées sont voisines ou même légèrement inférieures à ce qu'elles sont après l'arrêt des perfusions. C'est pour l'animal no 11 que l'augmentation des teneurs durant les premières périodes de perfusions est la plus élevée.

Les teneurs en histidine sont plus élevées dans le sang des trois chèvres lorsque ce composé leur est apporté par la perfusion que lors des périodes témoins du début et de la fin de l'expérience. Les valeurs des concentrations après l'arrêt des perfusions redeviennent voisines de celles obtenues en première période, sauf dans le cas de l'animal n 16 où elles restent relativement élevées.

Les teneurs en thréonine du sang des 3 chèvres tendent à diminuer entre les 
périodes 1 et 8 . Après l'arrêt des perfusions, elles redeviennent voisines ou même supérieures aux valeurs observées en période 1 pour les animaux $n^{\text {os }} 11$ et 16 . Une tendance similaire mais très atténuée semble exister en ce qui concerne les concentrations en phénylalanine entre les périodes 2 et 10 pour les chèvres nos 11 et 16 . Les concentrations en lysine ne semblent pas évoluer d'une manière semblable à celle de la thréonine; on remarque cependant, pour la chèvre $n^{0} 15$ une forte diminution entre la $1^{\text {re }}$ et la $2^{e}$ période.
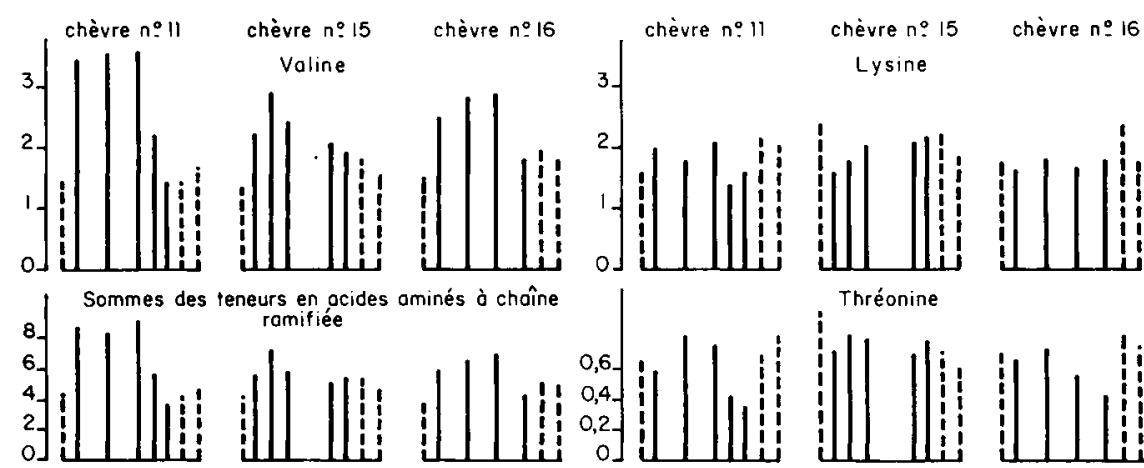

teneurs en acides aminés ò chaîne romifiée
romides en
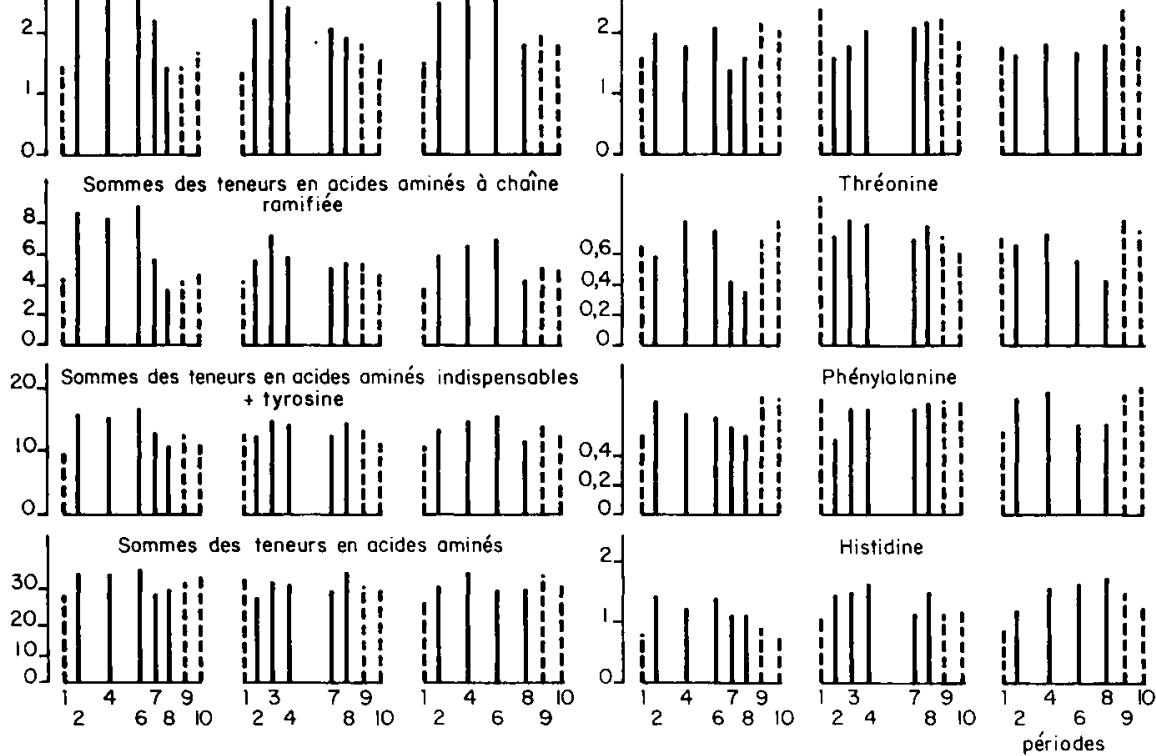

FIG. 1. - Evolution des teneurs en acides aminés libres du sang des chèvres au cours de l'expérience (mg p. $100 \mathrm{~g}$ de sang). Les traits continus correspondent aux périodes au cours desquelles des solutions d'acides aminés étaient perfusées.

Les sommes des teneurs en acides aminés libres ef amides du sang des chèvres $n^{0} 11$ et $n^{0} 16$ sont généralement plus faibles dans les échantillons correspondant à la période 1 que dans les autres prélèvements étudiés. Des valeurs relativement élevées sont observées durant les premières périodes de perfusion d'acides aminés, et durant les deux dernières périodes de l'expérience, alors que les perfusions avaient cessé. Des valeurs relativement faibles sont aussi observées durant les périodes au cours desquelles les teneurs en méthionine des solutions perfusées étaient les plus importantes. L'évolution de la somme des teneurs en acides aminés libres du sang de la chèvre no 15 est différente de celle qui correspond aux deux autres animaux. La valeur la plus faible est obtenue en période 2 et la plus élevée en période 8.

Les sommes des teneurs en acides aminés indispensables ( + tyrosine) évoluent de manière assez comparable à celles de l'ensemble des acides aminés. Toutefois, 
dans le cas de la chèvre no 15, l'écart entre les valeurs extrêmes est moins important que pour la somme des acides aminés et amides.

\section{Discussion.}

Cetfe expérience était destinée à nous renseigner sur les effets provoqués par l'apport direct aux tissus de méthionine, acide aminé particulièrement important chez le ruminant laitier (Rémond et al., 1971). Afin d'éviter l'interférence éventuelle d'autres facteurs limitants, nous avons ajouté aux solutions perfusées des quantités sensiblement constantes de valine, d'isoleucine, de leucine, qui sont prélevées dans le sang par la glande mammaire en quantités supérieures à celles qui sont incorporées dans le lait (Spires et al., 1975 ; Bickerstaffe, Annison et Linzell, 1974) et d'histidine ; celle-ci, présente en faible quantité dans les protéines bactériennes provenant du rumen et digérée dans l'intestin (Fauconneau et Pion, 1964), risquait d'être apportée en trop faible quantité aux tissus des animaux par l'alimentation.

L'augmentation de la teneur en matières azotées du lait pourrait être la conséquence de l'élévation relative des apports d'énergie par l'alimentation (Rémond et Journet, 1971). En effet, la consommation d'aliments n'a pas varié de manière importante en cours d'expérience alors que la production laitière a diminué ; la ration qui couvrait normalement les besoins des animaux en début d'expérience, apportait des quantités excédentaires d'azote et surtout d'énergie durant les dernières semaines. L'infusion de méthionine peut avoir aussi contribué à l'élévation du taux azoté du laił (Fisher, 1972).

Les valeurs observées pour les urémies et les glycémies sont normales et sont du même ordre de grandeur de celles obtenues à la suite d'expériences précédentes (Champredon, 1972).

Les teneurs en méthionine du sang des trois chèvres évoluent linéairement avec les « bilans apports-utilisation 》 définis précédemment (Champredon, 1972 ; tabl. 4). Ces «bilans » tiennent compte non seulement des quantités perfusées mais aussi de celles qui sont apportées à l'organisme par le tube digestif et incorporées dans les protéines du lait. La corrélation entre les teneurs et les « bilans » est hautement significative pour chaque chèvre. L'équation de la régression pour les trois animaux est :

$$
C=(0,27 \pm 0,03) B+0,51 ; r=0,87 ; P<0,001
$$

où $C$ représente la concentration de la méthionine en $\mathrm{mg} \mathrm{p} .100 \mathrm{~g}$ de sang et $B$ le «bilan apports-utilisation » en g par jour de méthionine. L'équation obtenue au cours d'une étude précédente (Champredon, 1972) avec deux chèvres laitières recevant par voie intraveineuse de la méthionine en présence de lysine et d'histidine étaif la suivante :

$$
\mathrm{C}=(0,33 \pm 0,07) \mathrm{B}+0,08 ; \mathrm{r}=0,88 ; \mathrm{P}<0,001 \text {. }
$$

L'écart entre les ordonnées à l'origine des droites correspondant aux deux équations est significatif $(P<0,001)$; il n'en est pas de même pour les pentes. Il semble donc que les conditions expérimentales et en particulier la nature des acides aminés présents dans la solution perfusée puissent modifier l'utilisation métabolique de la méthionine.

Les quantités d'acides aminés à chaîne ramifiée apportées chaque jour par la 
perfusion correspondent à celles qui sont excrétées dans $0,6 \mathrm{~kg}$ d'un lait de chèvre à 33 p. 1000 de matières azotées totales et à une augmentation d'environ 12 p. 100 de la fourniture de ces composés par le tube digestif aux tissus des animaux. Les teneurs en valine, isoleucine et leucine dans le sang sont considérablement augmentées à la suite des infusions et sont en grande partie responsables des variations de la somme des concentrations des acides aminés indispensables observées. La quantité d'histidine perfusée correspond à celle qui est présente dans $0,9 \mathrm{~kg}$ de laił et à une augmentation d'environ 20 p. 100 des apports du tube digestif. Le fait que l'élévation de l'histidinémie à la suite des perfusions soit proportionnellement plus faible que celles des concentrations en valine, isoleucine ef leucine pourrait signifier que ce composé est devenu limitant secondaire par suite d'une fourniture insuffisante aux tissus des animaux ou disparaît rapidement du sang (catabolisme, échange entre compartiments etc...).

La méthionine étant l'acide aminé le plus limitant pour les animaux laitiers, son apport en quantités croissantes à l'organisme fait diminuer les teneurs en certains autres acides aminés indispensables qui peuvent devenir limitants à leur tour. Cela semble être le cas de la thréonine et à un moindre degré de la phénylalanine mais les quantités de ces composés présentes dans le sang sont trop peu variables pour que les diminutions de teneurs soient significatives. Le problème est différent en ce qui concerne les trois acides aminés à chaîne ramifiée qui ont été ajoutés en quantités sensiblement constantes durant toutes les périodes de perfusion. La comparaison entre les feneurs observées dans le sang des chèvres $n^{\circ 8} 11$ et 16 lorsque la méthionine était perfusée en quantité supérieure à $2 \mathrm{~g}$ par jour avec celles qui correspondent à un apport de méthionine inférieur à cette valeur, montre une diminution significative des teneurs en valine, isoleucine $(P<0,005)$ ef leucine $(P<0,025)$.

En admettant que le besoin en méthionine des animaux est satisfait lorsque les apports intraveineux de ce composé provoquent une nette diminution des teneurs en certains autres acides aminés indispensables par suite d'une augmentation de leurs utilisations par les tissus, le besoin en méthionine de chèvres produisant entre 2 ef $3 \mathrm{~kg}$ de lait serait satisfait par un apport supplémentaire d'environ $2 \mathrm{~g}$ par jour de ce composé, ce qui correspond à un apport total de 4 à 4,5 g. Dans les conditions de la présente expérience, le besoin en méthionine de la chèvre no 15 dont la production laitière était toujours supérieure à $3 \mathrm{~kg}$ par jour n'était jamais satisfait durant les périodes de perfusion.

\section{Conclusion.}

La perfusion de méthionine aux chèvres laitières a permis de confirmer l'existence d'une relation linéaire entre les teneurs sanguines de ce composé et les « bilans apports-utilisation ". D'autre part, elle a eu pour conséquence l'abaissement dans le sang des concentrations en un certain nombre d'acides aminés indispensables, en particulier thréonine, valine, isoleucine et leucine. II est donc vraisemblable que la méthionine soit dans le cas de ces chèvres laitières, le premier acide aminé limitant ; elle serait suivie par l'histidine, la thréonine ef par les acides aminés à chaîne ramifiée. Il ne semble pas que la lysine soit un acide aminé limitant. 


\section{Références}

BICKERSTAFFE R., ANNISON E. F., LINZELL J. L., 1974. The metabolism of glucose, acetate, lipids and amino acids in lactating dairy cows. J. agric. Sci. Camb., 82, 71-85.

CHALUPA W., 1975. Rumen bypass and protection of proteins and amino acids. J. Dairy Sci., 58, 1198-1218.

CHAMPREDON C., 1972. Utilisation de l'aminoacidémie libre comme mesure de la satisfaction du besoin en certains acides aminés des chèvres laitières. Etude de quelques facteurs. Thèse de Doctorat de spécialité (Physiologie), Clermont-Ferrand.

CHAMPREDON C., PION R., THIVEND P., 1971. Composition en acides aminés des matières azotées du contenu duodénal du ruminant. $X^{\mathrm{e}}$ Congr. Int. Zoofech., Paris-Versailles, juillet 1971.

CLARK J. H., 1975. Lactational responses to postruminal administration of proteins and amino acids. J. Dairy Sci., 58, 1178-1197.

CLARKE E. M. W., ELLINGER G. M., PHILLIPSON A. T., 1966. The influence of diet on the nitrogenous components passing to the duodenum and through the lower ileum of sheep. Proc. Royal Soc., B, 166, 63-79.

DERRIG R. G., CLARK J. H., DAVIS C. L., 1974. Effect of abomasal infusion of sodium caseinate on milk yield, nitrogen utilisation and amino acid nutrition of the dairy cow. J. Nutr., 104, 151 159.

FAUCONNEAU G., PION R., 1964. Synthèse des protéines au niveau du rumen. Ann. Nutr. Aliment., 18, 86-97.

FERGUSON K. A., HEMSLEY J. A., REIS P. J., 1967. Nutrition and wool growth. The effect of protecting diefary protein from microbial degradation in the rumen. Austr. J. Sci., 30, 215-217.

FISHER L. J., 1972. Response of lactating cows to the intravenous infusion of amino acids. Can. J. anim. Sci., 52, 377-384.

LEROY F., ZELTER S. Z., FRANÇOIS A. C., 1964. Protection des protéines alimentaires contre la désamination bactérienne au niveau du rumen. C. R. Acad. Sci. Paris, 259, 1592-1595.

PAWLAK M., PION R., 1968. Influence de la supplémentation des protéines du blé par des doses croissantes de lysine sur la teneur en acides aminés libres du sang et du muscle du rat en croissance. Ann. Biol. anim. Bioch. Biophys., 8, 517-530.

REMOND B., CHAMPREDON C., DECAEN C., PION R., JOURNET M., 1971. Influence d'un apport de DL-Méthionine à des vaches au début de la lactation sur la production laitière et la composition du sang. Ann. Biol. anim. Bioch. Biophys., 11, 455-469.

REMOND B., JOURNET M., 1971. Alimentation des vaches laitières avec des rations à forte proportion d'aliments concentrés. I. Quantités ingérées et production laitière. Ann. Zootech., 20, 169-184.

SPIRES H. R., CLARK J. H., DERRIG R. G., DAVIS C. L., 1975. Milk production and nitrogen utilisation in response to postruminal infusion of sodium caseinate in lactating cows. J. Nutr., 105, 1111-1121. 